\title{
Modeling the thermal stability of precursor nanoparticles in zeolite synthesis
}

\author{
MIGUEL JORGE†, SCOTT M. AUERBACH $§$ and PETER A. MONSON§ \\ $\dagger$ REQUIMTE, Faculdade de Ciências, Universidade do Porto, Rua do Campo Alegre 687, 4169-007 Porto, Portugal \\ Email: miguel.jorge@fc.up.pt \\ $\ddagger$ Department of Chemistry, University of Massachusetts, Amherst, MA 01003 USA \\ Email: auerbach@chem.umass.edu \\ $\S$ Department of Chemical Engineering, University of Massachusetts, Amherst, MA 01003 USA \\ Email: monson@ecs.umass.edu \\ (Received 00 Month 200x; In final form 00 Month 200x)
}

We have modelled the thermal stability of silica nanoparticles commonly observed as precursors in the synthesis of zeolites. We performed canonical Monte Carlo and parallel tempering simulations on a lattice model that describes the self-assembly of nanoparticles under conditions at which they are observed experimentally. The effect of heating on the relative stability of the phases of the model was analysed by running simulations at various temperatures. At low temperature, the model yields a metastable multi-particle phase with a characteristic size distribution, which is separated by an energy barrier from the true equilibrium phase, a dense silica solid. As temperature increases, the system enters a transition region and eventually reaches the bulk phase. This transition is reminiscent of the experimentally observed transition from nanoparticles to zeolite. The transition temperature scales with the inverse of the system volume approaching an asymptotic value for large system sizes. This indicates the transition temperature is a reproducible macroscopic property of the system. The transition temperature in the model is within the range of temperatures at which nanoparticles form zeolite crystals in experiments.

Keywords: Monte Carlo simulation, lattice, metastability, silica, nucleation

\section{Introduction}

Zeolites are crystalline alumino-silicates with a regular nanoporous network, and are thus employed in many industrial processes such as catalysis and separations [1]. The range of applications of zeolites has been expanding rapidly, and now includes hydrocarbon conversions [2], optical electronics [3], bio-implants [4] and enantioselective separations [5]. Due to this growing interest, it has become 
crucial, from both a scientific and a technological perspective, to develop a detailed understanding of the synthesis of these materials. The ability to control the synthesis of zeolites would not only allow for optimisation of the above processes, but would also permit the design of new materials tailored for specific applications. This has led to many experimental and theoretical studies of zeolite synthesis [5,6]. More recently, molecular modelling efforts have also been directed toward this area of research, yielding important insights [7]. In the past decade, evidence has pointed to an important role played by silica nanoparticles in zeolite formation [8-13]. We have recently developed a lattice model to simulate the formation and properties of these nanoparticles [14], and have obtained good agreement with experimental observations on the variation of nanoparticle size with synthesis conditions [15]. Here, we expand on this work by studying the effect of temperature on the stability and size of the nanoparticles for the model.

The synthesis of silicalite-1, the pure-silica form of zeolite ZSM-5 (framework code MFI), has been one of the most well studied cases. This material was first reported by Flanigen et al. [16] and is composed of parallel straight cylindrical channels, connected by cylindrical zigzag channels [17]. One may synthesise silicalite-1 crystals starting from a clear solution of tetraethyl orthosilicate (TEOS), tetrapropylammonium hydroxide (TPAOH) and water. Heating of this solution to $\sim 100^{\circ} \mathrm{C}$ for a few hours yields high-quality crystals of silicalite-1 [18]. After this step, the channel intersections are occupied by tetrapropylammonium cations (TPA), with the four propyl groups extending into the adjacent channels. The TPA can be removed by calcination to produce a nanoporous crystalline solid.

The mechanism of zeolite formation from solution is still poorly understood. One of the facts that is generally agreed upon is the presence of an apparently stable suspension of nanometer-sized silica particles at room temperature, prior to the observation of any zeolite crystals [8-13]. The structure of these nanoparticles and their precise role in the zeolite synthesis are still the subject of debate $[8,10-12,19]$. Recently, however, substantial progress has been made in understanding the processes by which silica nanoparticles are formed and stabilised in solution at room temperature. A critical aggregation concentration of $\mathrm{SiO}_{2}$ has been identified, below which the solution contains primarily silica monomers and small oligomers, and above which nanoparticles spontaneously self-assemble [15]. In situ small-angle X-ray scattering (SAXS) and small-angle neutron scattering (SANS) studies have shown that the particles are composed of a core of mostly amorphous silica, which is negatively charged at the surface, surrounded by a shell of organic cations $[15,20]$. Nanoparticle size was seen to be independent of the type of alkyl-ammonium cation used, and particles were even observed in silica solutions containing only small inorganic cations [20].

Monte Carlo (MC) simulations of our lattice model have qualitatively captured all of these experimental observations [14]. Furthermore, the model has yielded several predictions that have since been confirmed from analyses of experimental data.

Rimer et al. [21] have characterised solutions with different concentrations of TPAOH and TPABr to distinguish between the effects of $\mathrm{pH}$ and TPA concentration. These experiments have confirmed our theoretical prediction that nanoparticle size is controlled by the initial $\mathrm{pH}$ of the solution, rather than by the cation concentration. Other studies [21,22] have supported our hypothesis that, following a rapid initial period of monomer aggregation to form many small clusters, the silica particles grow by Ostwald ripening, rather than by nanoparticle aggregation. In a recent paper, Davis et al. [19] have presented experimental results on nanoparticle evolution at room temperature over a long period of time. These authors have observed the formation of zeolite crystals after a prolonged nucleation stage, establishing conclusively that the nanoparticles are a metastable phase as put forward in our simulation work [14]. Both our simulations and a subsequent experimental study [23] suggest that the particles are stabilised at least partially by electrostatic interactions at the surface, caused by the presence of the cation shell.

From the experimental and theoretical studies mentioned above, a picture has been emerging in which the nanoparticles are amorphous 
silica entities that serve as a source of material for the growth of zeolite crystals (see [24] and references therein). Formation of zeolite crystals from nanoparticles occurs upon heating of the solution, making temperature a key variable to be studied. Indeed, it is known that silica nanoparticles exhibit irreversible structural changes at high temperatures [15]. In a recent study, Rimer et al. have examined in more detail the effect of temperature on a solution of TEOS and TPAOH [21]. They have shown that the nanoparticles increase in size with temperature, growing by Ostwald ripening, but still retain their core-shell structure. However, they have also concluded that at high temperatures TPA cations (and possibly water) are incorporated into the nanoparticle core, the composition of which starts to become more zeolite-like. In spite of these insights, a molecular-level understanding of the nature of these changes, as well as their importance in the zeolite nucleation and growth processes, is still lacking. In this paper, we address this issue by examining the effect of temperature on our previously developed lattice model for the formation of silica nanoparticles [14]. We analyse the relative stability of the phases of the model at different temperatures, and look more closely at the transition from the nanoparticle phase to a phase of bulk silica. A parallel is drawn between our model and the real silica solution, which can provide insight into the problem of zeolite crystal growth.

The paper is organized as follows. Section 2 presents a brief description of the model we have developed for the TEOS-TPAOH-water system, and of the simulation techniques employed. Section 3 presents the results obtained from the simulations, analysing the behaviour of the model for a wide temperature range. Finally, we present our conclusions in Section 4.

\section{Model and Methods}

We have used a lattice model to study the formation and stability of silica nanoparticles at a range of temperatures. This model, and the simulation techniques used here, have been described in detail elsewhere [14], so we will limit ourselves to a short outline of the most important aspects.

Lattice models have several advantages relative to more fine-grained approaches, most importantly the possibility to simulate large systems for long times. This feature is particularly important for studying nanoparticle formation, since large system sizes are needed to obtain statistically meaningful collections of nanoparticles, and long simulations are necessary to observe the spontaneous formation and evolution of nanoparticles. Off-lattice atomistic approaches previously used to model silica polymerisation [25-30] are currently restricted to small system-sizes and/or short times, due to limitations in available computer power. On the other hand, lattice models have been able to provide qualitative information about complex systems such as surfactant-oil-water micellar solutions [31-35] and surfactant-silica solutions [36].

Our first step in coarse graining is to replace atomistic detail in favour of "united atom" representations, where each molecule is represented by a single site, rather than by an explicit representation of all its atoms. In addition, we divide three-dimensional space into a simple cubic lattice, with every lattice site occupied by a single species. This implies an increase in the coordination number of silica from 4 to 6 , an approximation that will be relaxed in a forthcoming publication.

In an alkaline solution composed of water, $\mathrm{TPA}, \mathrm{Si}(\mathrm{OH})_{4}$ and $\mathrm{OH}^{-}$, the main reactions taking place (written in a generic form) are silica condensation/hydrolysis:

$$
\mathrm{R}-\mathrm{Si}-\mathrm{OH}+\mathrm{R}^{\prime}-\mathrm{Si}-\mathrm{OH} \rightleftharpoons \mathrm{R}-\mathrm{Si}-\mathrm{O}-\mathrm{Si}-\mathrm{R}^{\prime}+\mathrm{H}_{2} \mathrm{O}
$$


and silica deprotonation:

$$
\mathrm{R}-\mathrm{Si}-\mathrm{OH}+\mathrm{OH}^{-} \rightleftharpoons \mathrm{R}-\mathrm{Si}-\mathrm{O}^{-}+\mathrm{H}_{2} \mathrm{O}
$$

The first reaction results in a multiplicity of silicate species (dimers, trimers, rings, etc.). Instead of enumerating all of these species as separate components in our model, which would quickly become unmanageable, we consider only silica monomers and structures derived from them. If during the simulation two monomers move next to each other, we say that a dimer has formed. If a third monomer moves next to the previous two, a trimer is formed, and so on. The energy attributed to this move represents the internal energy of the condensation reaction (1) (a reaction energy, not a bond association energy). Both computational [37] and experimental [38] studies have shown that the silica condensation reaction is exothermic, which means that the interaction energy in our model should be attractive. Such an attraction drives the clustering of monomers, while entropy tends to keep monomers dissolved in solution. In what follows, all energies are divided by the absolute value of the attraction between two neutral silica monomers, and are denoted by $\varepsilon_{i}^{*}$.

The second reaction is accounted for implicitly by distinguishing between neutral and ionised silica monomers (denoted hereafter by $\mathrm{S}_{\mathrm{N}}$ and $\mathrm{S}_{\mathrm{I}}$, respectively). We assume that the silica deprotonation reaction reaches equilibrium at the start of the simulation; this assumption is supported by reaction ensemble simulations in our earlier work [14]. We note that we have not considered the possiblity of further deprotonations of anionic monomers. Even though doubly ionised monomers $\left(\mathrm{Si}(\mathrm{OH})_{2} \mathrm{O}_{2}^{2-}\right)$ have been shown to exist at high enough $\mathrm{pH}$ [38-40], they are relatively unreactive in polymerisation [41], and would thus add unnecessary complexity to our model. The mole fractions of all components $\left(\mathrm{S}_{\mathrm{N}}, \mathrm{S}_{\mathrm{I}}\right.$, TPA and water) at the start of the simulation can be calculated as described in detail in Ref. [14]. In this paper, we are mainly concerned with the effect of temperature on silica nanoparticles, since the effect of other experimental control variables ( $\mathrm{pH}$, concentration of TEOS, concentration of TPA, cation size) has been studied in detail previously [14]. Thus, all simulations presented here correspond to the same set of experimental mole ratios, namely $40 \mathrm{SiO}_{2}: 9$ TPAOH: $9500 \mathrm{H}_{2} \mathrm{O}: 160$ Ethanol.

Each lattice site is occupied by one of the four components: neutral silica monomer $\left(\mathrm{S}_{\mathrm{N}}\right)$, anionic silica monomer $\left(\mathrm{S}_{\mathrm{I}}\right)$, template molecule (TPA), solvent molecule (W). Without loss of generality, we set all interactions with solvent to zero; as such, we do not account explicitly for the dynamics of solvent particles, but rather view them as vacancies in the lattice. The system configurations displayed below omit solvent particles for clarity.

In addition to distinguishing between neutral and ionised silica monomers, we also take into account the orientation of ionised silica monomers to reflect the localized charge in $\mathrm{Si}(\mathrm{OH})_{3} \mathrm{O}^{-}$groups. To represent this effect, a pointer variable is assigned to each lattice site occupied by an $\mathrm{S}_{\mathrm{I}}$. This variable "points" to one of the neighbours of that site, to indicate the orientation of the negatively charged oxygen (denoted by $\mathrm{S}_{\mathrm{I}}^{-}$). The remaining neighbours interact with a neutral hydroxyl group (denoted simply by $\mathrm{S}_{\mathrm{I}}$ ). As a result of these choices, one must set an additional 5 interaction energies between silicate species alone, relative to $\left|\varepsilon_{S_{N} S_{N}}\right|$. Please see Ref. [14] for a detailed discussion of these interaction parameters.

The large size of the TPA cation, relative to the size of the solvent and silica monomers, must also be taken into account. This is done in a simplified fashion, by including a nearest-neighbour repulsion shell around each TPA molecule. Provided this repulsion is strong enough, all of the first-neighbour sites of each TPA will remain vacant, thus effectively increasing its collision diameter. To eliminate configurations in which two of these large molecules sit adjacently to each other in a diagonal arrangement, one must also include a 
second-neighbour repulsion between TPA molecules. Incorporation of this repulsion shell implies that the attraction between TPA and $\mathrm{S}_{\mathrm{I}}^{-}$, as well as the pointer variable, must be extended to a second-neighbour level.

Following the above description, the lattice model Hamiltonian may be expressed as a sum over all contacts between components on the lattice:

$$
E=\frac{1}{2} \sum_{i=1}^{4} \sum_{j=1}^{4}\left(\mathcal{N}_{i j}^{\mathrm{FN}} \varepsilon_{i j}^{\mathrm{FN}}+\mathcal{N}_{i j}^{\mathrm{SN}} \varepsilon_{i j}^{\mathrm{SN}}\right)
$$

where $\mathcal{N}_{i j}$ is the total number of neighbour contacts between components $i$ and $j, \varepsilon_{i j}$ is the energy of interaction between those species, and superscripts FN and SN denote first- and second-neighbour interactions. The summations run only over 4 components, not 5 , because the solvent molecules are considered as vacancies (all interaction energies are zero). Tables 1 and 2 show the first- and second-neighbour reduced interaction parameters for the model, respectively.

[insert Table 1 about here]

[insert Table 2 about here]

In this formulation, the state of the model is fully specified by setting the system composition (obtained from the experimental mole ratios, as described above) and the reduced temperature, defined here as $T^{*}=k_{\mathrm{B}} T /\left|\varepsilon_{\mathrm{S}_{\mathrm{N}} \mathrm{S}_{\mathrm{N}}}\right|$, where $k_{\mathrm{B}}$ is Boltzmann's constant. In order to obtain a correspondence between the model (reduced) and real (absolute) temperature scales, one must determine, at least approximately, the value of $\varepsilon_{\mathrm{S}_{\mathrm{N}} \mathrm{S}_{\mathrm{N}}}$. By simulating the solubility of amorphous silica at neutral $\mathrm{pH}$, we have calibrated this effective interaction to a value of $-2.5 \mathrm{kcal} / \mathrm{mol}$ [14]. This is close to the value of $-2.2 \mathrm{kcal} / \mathrm{mol}$ obtained from $a b$ initio calculations of silica dimerisation [37]. Using the value obtained from our calibration, room temperature corresponds roughly to $T^{*}=0.24$.

We have performed simulations at several temperatures using Monte Carlo in the canonical ensemble $(N V T)$, on a simple cubic lattice with periodic boundary conditions in all three cartesian directions. Two types of MC moves were implemented: a swap move, consisting of an exchange in position between two molecules located on different sites of the lattice; and a rotation of the pointer variable placed on the anionic silica sites. The new pointer orientation is chosen at random from all 12 possible values (one for each second neighbour). The trials are accepted or rejected based on the Boltzmann factor associated with each configurational change [42].

Except where noted, each $N V T$ MC run was started from a random initial configuration, in a cubic simulation box of side $L$, and allowed to equilibrate for at least 1 million sweeps. A sweep is defined as $N_{\text {occ }}$ attempted moves plus $N_{\text {occ }}$ attempted rotations, where $N_{\text {occ }}$ is the number of occupied sites on the lattice (excluding solvent). Outputs of each run are the average energy of the system, the average cluster size and cluster size distribution, as well as snapshots of configurations. Cluster size distributions were computed by implementing the Hoshen-Kopelman cluster-counting algorithm [43]. Two silica monomers are considered to belong to the same cluster if they are connected by a single lattice bond.

We have also employed the parallel tempering (PT) simulation technique $[44,45]$ to improve the efficiency of equilibration. It is implemented by performing $M$ separate $N V T$ simulations at different temperatures, each replica equilibrating independently by virtue of conventional MC moves. After a preset number of these moves, a replica swap, consisting of an exchange of the complete configuration between two replicas running at adjacent temperatures, is attempted. The replica exchange trial is accepted with a 
probability given by:

$$
p=\min \left\{1, \exp \left[\left(\frac{1}{k_{\mathrm{B}} T_{j}}-\frac{1}{k_{\mathrm{B}} T_{i}}\right)\left(E_{j}-E_{i}\right)\right]\right\}
$$

where $E_{i}$ is the total energy of configuration $i$. Decreasing the spacing between replicas, by increasing $M$, decreases the energy difference in eq (4), thus increasing the acceptance ratio. However, the computer time increases proportionally to $M$. Therefore, $M$ must be as small as possible, while still ensuring adequate sampling of the desired temperature range. We have used an iterative procedure, described in detail elsewhere [14], to find the ideal value of $M$ and the optimum spacing between replicas.

\section{Results and Discussion}

The control variables in an experiment of silicalite- 1 synthesis from clear solution are the molar ratios of the species present, the nature of the cation and the temperature. In our previous work [14], we have examined closely the effect of the first two, showing that the model yields results in good qualitative agreement with SAXS and SANS measurements of nanoparticle size by Fedeyko et al. [15]. In this paper, the type of cation and the species concentrations are kept fixed, while the effect of changing the system temperature is examined in more detail. The concentrations are chosen so that a stable population of silica nanoparticles is observed at room temperature in both the experiments and the simulations, i.e., we are operating above the critical silica concentration for spontaneous nanoparticle formation $[14,15]$.

Figure 1 shows a plot of average cluster size as a function of reduced temperature, obtained from $N V T$ and PT simulations for two different lattice sizes. In the parallel tempering curves (triangles), one can distinguish two phases of silica. All replicas below $T^{*}=0.41$ equilibrate to a single, very large silica cluster that captures most of the silica in the system. This large cluster corresponds to a bulk solid phase in equilibrium with a dilute solution. As such, the size of this bulk solid depends on system size, increasing proportionally to $L^{3}$. Its size decreases with temperature due to increased silica solubility, until it undergoes a "melting" transition at $T^{*}=0.41$, to yield a random distribution of monomers and a few small oligomers.

The results from $N V T$ simulations (circles) have been averaged over five independent runs, with different initial conditions. One can identify four distinct regions in the $N V T$ curves: a low-temperature region (roughly $0.24<T^{*}<0.3$ ); a transition region (between 0.3 and either 0.34 or 0.35 , depending on the lattice size); a bulk silica region (between 0.34 or 0.35 and 0.41 ); and a liquid solution region (for $T^{*}>0.41$ ). The bulk silica and liquid solution regions are equivalent to those observed in the PT simulations.

[insert Figure 1 about here]

At low temperature, the average cluster size is significantly below the parallel tempering results, and is independent of system size. In this region, all runs yield a population of small negatively charged clusters composed of a core of silica and a layer of TPA cations at the surface. This phase is metastable and corresponds to the silica nanoparticle phase observed in experiments at room temperature [8-13,15], characterised in our previous work [14]. During a given simulation run, practically all neutral silica monomers quickly coalesce to form a large number of small clusters, which subsequently grow by Ostwald ripening (dissolution of smaller clusters, which provide material for the growth of larger ones [46]). At a certain point, however, particle growth comes to a halt - the 
protective layer of TPA prevents further growth of the particles, and thus contributes to free energy barriers keeping the nanoparticles metastable. Using parallel tempering, one is able to surmount these barriers and reach the true equilibrium state of the model, bulk silica. In contrast to the PT curves, the average cluster size in the metastable region increases slightly with temperature, which is once again due to an increase in silica solubility. While in the bulk phase higher solubility simply means more dissolved silica (and hence less silica in the solid phase), in the metastable phase, it increases the tendency for Ostwald ripening, hence increasing the average cluster size at which this process stops. This increase in particle size with temperature, occurring by Ostwald ripening, is in agreement with experimental observations [21].

When $T^{*}$ is increased further, another process emerges — some of the $N V T$ runs start to reach the bulk silica phase. In this transition region, thermal fluctuations are occasionally sufficient to overcome the energy barriers stabilising the nanoparticles. The proportion of individual runs that reach equilibrium increases with $T^{*}$, causing a steep increase in average cluster size with temperature, widening the statistical error in the average cluster size, and producing a deviation between the curves for different lattice sizes. This last effect stems from the fact that single bulk clusters are larger for the larger lattice, and hence weigh the average cluster size toward higher values. At sufficiently high temperature, all runs reach the maximum cluster size imposed by the lattice dimensions, and the $N V T$ curves coincide with the respective PT plots.

The data shown in Figure 1 suggest that the transition temperature from nanoparticles to bulk silica depends on the system size, since this transition happens at lower $T^{*}$ for the smaller lattice. To investigate this, we have located transition temperatures for other lattice sizes $(\mathrm{L}=60,80$ and 140$)$ through an interative bracketing technique. The transition temperature is defined as the value of $T^{*}$ for which all five independent runs reach the bulk phase. Calculation of full curves analogous to those in Figure 1 for each lattice size was not attempted because these are computationally very intensive.

[insert Figure 2 about here]

The transition temperature scales as $L^{-3}$ as shown in Figure 2. This is consistent with the finite size scaling observed for first order transitions in previous Monte Carlo simulations [47,48]. Here, however, we have a transition from a metastable state to an equilibrium one rather than between two equilibrium states. Extrapolation to infinite system size yields a transition temperature of $T^{*}=0.368$.

Our results are consistent with the transition being a reproducible macroscopic phenomenon.

By averaging over 60 statistically independent $N V T$ runs, we obtained cluster size distributions (CSD) for several representative temperatures (shown in Figure 3 ). The CSD at the lowest temperature $\left(T^{*}=0.24\right)$ shows a peak at around 300 silica units and a tail extending to about 2000 units. This shape is characteristic of the metastable silica nanoparticle phase, and has been shown to be reproducible and independent of system size for sufficiently large systems [14]. The mean cluster size at $T^{*}=0.24$ ( 500 Si units) is in reasonable agreement with experiment at these conditions [20,23], which is remarkable given the simplicity of this model. Increasing $T^{*}$ to 0.30 shifts the main peak to higher cluster sizes and pushes the tail to about 3200 silica units. As explained above, these changes result from Ostwald ripening progressing to larger particle sizes. Nevertheless, the system at $T^{*}=0.30$ remains in the metastable region; the CSD retains the same unimodal character as the one at $T^{*}=0.24$. It is noteworthy, however, that some configurations reach cluster sizes already very close to the maximum size allowed $(\approx 3600$ units for this lattice size), suggesting that thermal fluctuations are becoming large enough and frequent enough to cross the free energy barriers.

The CSD at $T^{*}=0.33$ shows a substantially different picture. The distribution is now bimodal, with a first peak spread out over a very 
wide range of sizes, and a second narrow peak at 3250 units. The latter peak corresponds to the largest possible cluster at this system size, and shows that a number of runs have reached true equilibrium. The absence of a clearly defined peak at small cluster sizes signals a breakdown of the metastable nanoparticle phase. At this temperature, the system is in the middle of the transition region; the average cluster size is determined by the fraction of configurations that reach the bulk phase (see figure 1). Finally, the CSD at the highest temperature $T^{*}=0.35$ shows a single narrow peak at 3000 units. This corresponds to the bulk silica phase, which at these conditions is accessed by all NVT runs.

[insert Figure 3 about here]

It is instructive to analyse the evolution of the average cluster size during individual $N V T$ runs, since this provides information on the nature of the transition from metastable nanoparticles to bulk silica. Figure 4 shows such plots for typical runs at the same four representative temperatures studied in figure 1 . The configurations at the two lowest temperatures $\left(T^{*}=0.24\right.$ and 0.30$)$ remain frozen once they reach a given particle size, since they are still in the metastable region. As expected, the curve for $T^{*}=0.30$ yields a higher average cluster size. In contrast, the average cluster size for $T^{*}=0.35$ increases very rapidly, reaching the maximum value commensurate with the system size very early in the simulation.

The plot at $T^{*}=0.33$ shows the most interesting behaviour: it seems to settle in a plateau of about 1000 silica units, but then jumps suddenly to the bulk phase after about $1.3 \times 10^{6}$ sweeps. We note that not all runs at this $T^{*}$ show identical behaviour - some remain in the metastable state while others go straight to the bulk phase. Thus, the average of several runs will lie somewhere between the value for the silica nanoparticles and that of bulk silica (see Figure 1). Furthermore, even though the transition from metastability to equilibrium may be abrupt in a particular run, averaging over several runs results in a smooth transition over a temperature range, rather than at a single value of $T^{*}$.

[insert Figure 4 about here]

To better illustrate the evolution of the run at $T^{*}=0.33$, we show in figure 5 snapshots of configurations obtained at several stages of the simulation. Very early on, the silica condenses to form several small clusters (figure 5b), and the system practically runs out of free $\mathrm{S}_{\mathrm{N}}$ monomer. Ostwald ripening then progresses for several sweeps, until the system stabilises in a configuration containing three silica nanoparticles (figure 5c). This configuration is quite stable, and remains practically unchanged for almost one million sweeps (figure 5d). At this point, however, a fluctuation triggers dissolution of the particle closest to the bottom of the simulation cell (figure 5e). Very soon afterwards, the topmost cluster also dissolves, yielding a single cluster of bulk silica (figure 5f). The shift from a 3-cluster phase to the bulk phase happens over the course of only $1.3 \times 10^{5}$ sweeps.

[insert Figure 5 about here]

\section{Conclusions}

We have explored a lattice model developed previously to explain spontaneous nanoparticle formation during clear-solution synthesis of zeolites. In particular, we have studied the effect of heating on the relative stability of the phases of the model. At low temperature, the model exhibits a metastable phase composed of multiple silica clusters, and which possesses a characteristic unimodal size distribution. 
The true equilibrium phase, however, is a single large cluster of silica, but this phase can be accessed at low temperatures only by employing special simulation techniques such as parallel tempering. The multicluster phase is separated from the bulk phase by a free energy barrier, associated with a protective layer of TPA cations formed at the particle surface. At low temperatures, the average cluster size of the metastable phase increases mildly with $T^{*}$, since increased mobility of the protective layer allows Ostwald ripening to produce larger nanoparticles.

As temperature is increased further, thermal fluctuations become large enough to overcome the energy barrier separating the two phases. In this transition region, some individual $N V T$ runs show abrupt shifts from multiple clusters to bulk, and cluster size distributions become bimodal. Finally, at high enough temperature, all runs reach equilibrium and the average cluster sizes from $N V T$ and parallel tempering coincide. The precise value of $T^{*}$ at which these two curves merge scales inversely with system volume and tends asymptotically to a finite temperature.

It is now possible to establish a link between the behaviour of our lattice model and that of the zeolite synthesis process. The metastable phase present in the model in the low temperature region (corresponding to a room temperature aqueous solution of TPAOH and TEOS) has been identified with the silica nanoparticles observed in experiments [14]. The equilibrium state of the model may be identified with a thermodynamically stable solid silica phase, which in experiments may be template-filled silicalite-1 zeolite. The transition between these two states in our model takes place at temperatures close to those at which nanoparticles are heated in experiments to produce zeolite crystals (in our model, $T^{*}=0.368$ corresponds roughly to $180^{\circ} \mathrm{C}$ ). Furthermore, particle growth at high temperatures proceeds by Ostwald ripening, which also agrees with experimental observations [21]. Therefore, the results of this work support a mechanism in which zeolite crystals grow at the expense of nanoparticles, since these are no longer stable and can provide a source of material for the growth process. Of course, the present lattice model does not reproduce a crystalline zeolite phase, so the model nanoparticles simply feed the growth of a dense silica solid. Further work using more realistic models (on- or off-lattice) is necessary to realize the computational self-assembly of zeolites.

\section{Acknowledgments}

The authors thank the National Science Foundation for their generous support through a Nanoscale Interdisciplinary Research Team (NIRT) grant (CTS-0103010).

\section{References}

[1] S. M. Auerbach, K. A. Carrado, and P. K. Dutta, editors, Handbook of Zeolite Science and Technology, Dekker Inc.: New York, 2003.

[2] T. F. Degnan, G. K. Chitnis, and P. H. Schipper, Microporous Mesoporous Mater. 35, 245 (2000).

[3] Y. Wada, T. Okubo, M. Ryo, T. Nakazawa, Y. Hasegawa, and S. Yanagida, J. Am. Chem. Soc. 122, 8583 (2000).

[4] S. I. Zones and M. E. Davis, Curr. Opin. Solid State Mat. Sci. 1, 107 (1996).

[5] M. E. Davis and R. F. Loвo, Chem. Mater. 4, 756 (1992).

[6] C. S. Cundy and P. A. Cox, Chem. Rev. 103, 663 (2003).

[7] S. M. Auerbach, M. H. Ford, and P. A. Monson, Curr. Opin. Colloid Interface Sci. 10, 220 (2005). 
[8] B. J. Schoeman, Microporous Mesoporous Mater. 22, 9 (1998).

[9] J. N. Watson, L. E. Iton, R. I. Keir, J. C. Thomas, T. L. Dowling, and J. W. White, J. Phys. Chem. B 101, 10094 (1997).

[10] P. E. A. de Moor, T. P. M. Beelen, and R. A. van Santen, J. Phys. Chem. B 103, 1639 (1999).

[11] R. Ravishankar, C. E. A. Kirschhock, P. Knops-Gerrits, E. J. P. Feijen, P. J. Grobet, P. Vanoppen, F. C. de Schryver, G. Miene, H. Fuess, B. J. Schoeman, P. A. Jacobs, and J. A. Martens, J. Phys. Chem. B 103, 4960 (1999).

[12] D. D. Kragten, J. M. Fedeyko, K. R. Sawant, J. D. Rimer, D. G. Vlachos, R. F. Lobo, and M. Tsapatsis, J. Phys. Chem. B 107, $10006(2003)$.

[13] S. Yang, A. Navrotsky, D. J. Wesolowski, and J. A. Pople, Chem. Mater. 16, 210 (2004).

[14] M. Jorge, S. M. Auerbach, and P. A. Monson, J. Am. Chem. Soc. 127, 14388 (2005).

[15] J. M. Fedeyko, J. D. Rimer, R. F. Lobo, and D. G. Vlachos, J. Phys. Chem. 108, 12271 (2004).

[16] E. M. Flanigen, J. M. Bennett, R. W. Grose, J. P. Cohen, R. L. Patton, R. M. Kirchner, and J. V. Smith, Nature 271, $512(1978)$.

[17] R. F. Loвo, Handbook of Zeolite Science and Technology, chapter Introduction to the Structural Chemistry of Zeolites (3), p. 65, Dekker Inc.: New York, 2003.

[18] B. J. Schoeman and O. Regev, Zeolites 17, 447 (1996).

[19] T. M. Davis, T. O. Drews, H. Ramanan, C. He, J. Dong, H. Schnablegger, M. A. Katsoulakis, E. Kokkoli, A. V. McCormick, R. L. Penn, and M. Tsapatsis, Nature Mater. 5, 400 (2006).

[20] J. M. Fedeyko, D. G. Vlachos, and R. F. Lobo, Langmuir 21, 5197 (2005).

[21] J. D. Rimer, D. G. Vlachos, and R. F. Lobo, J. Phys. Chem. B 109, 12762 (2005).

[22] J. L. Provis and D. G. Vlachos, J. Phys. Chem. B 110, 3098 (2006).

[23] J. D. Rimer, R. F. Lobo, and D. G. Vlachos, Langmuir 21, 8960 (2005).

[24] J. D. Rimer, J. M. Fedeyko, D. G. Vlachos, and R. F. Lobo, Chem. Eur. J. 12, 2926 (2006).

[25] B. P. Feuston and S. H. Garofalini, J. Phys. Chem. 94, 5351 (1990).

[26] S. H. Garofalini and G. Martin, J. Phys. Chem. 98, 1311 (1994).

[27] P. Vashishta, R. K. Kalia, J. P. Rino, and I. Ebbsjö, Phys. Rev. B 41, 12197 (1990).

[28] N. Z. RaO and L. D. Gelb, J. Phys. Chem. 108, 12418 (2004).

[29] M. G. Wu and M. W. Deem, J. Chem. Phys. 116, 2125 (2002).

[30] Y. Tu, J. Tersoff, G. Grinstein, and D. Vanderbilt, Phys. Rev. Lett. 81, 4899 (1998).

[31] R. G. Larson, J. Chem. Phys. 91, 2479 (1989).

[32] A. D. Mackie, A. Z. Panagiotopoulos, and I. Szleifer, Langmuir 13, 5022 (1997).

[33] B. Widom, J. Chem. Phys. 84, 6943 (1986).

[34] K. Chen, C. Ebner, C. Jayaprakash, and R. Pandit, Phys. Rev. A 38, 6240 (1988).

[35] H. J. Woo, C. Carraro, and D. Chandler, Faraday Discuss. 104, 183 (1996).

[36] F. R. Siperstein and K. E. Gubbins, Langmuir 19, 2049 (2003).

[37] C. R. A. Catlow, D. S. Coombes, B. Slater, D. W. Lewis, and J. C. G. Pereira, Handbook of Zeolite Science and Technology, chapter Modeling Nucleation and Growth in Zeolites (4), p. 91, Dekker Inc.: New York, 2003.

[38] R. K. ILER, The Chemistry of Silica: solubility, polymerization, colloid and surface properties, and biochemistry, Wiley: New York, 1979 . 
[39] C. J. Brinker and G. W. Scherer, Sol-gel Science: the physics and chemistry of sol-gel processing, Academic Press: San Diego, 1990.

[40] J. ŠEFČÍK and A. V. MCCormick, AIChE J. 43, 2773 (1997).

[41] S. D. Kinrade and T. W. Swaddle, Inorg. Chem. 27, 4259 (1988).

[42] D. Frenkel and B. Smit, Understanding Molecular Simulation: from algorithms to applications, Academic Press: San Diego, 1996.

[43] J. Hoshen and R. Kopelman, Phys. Rev. B 14, 3438 (1976).

[44] K. Hukushima and K. Nemoto, J. Phys. Soc. Jpn. 65, 1604 (1996).

[45] D. A. Kofke, J Chem. Phys. 117, 6911 (2002).

[46] L. Ratke and P. W. VoonheEs, editors, Growth and coarsening: ostwald ripening in material processing, Springer-Verlag Telos: New York, 2002

[47] K. Binder and D. P. Landau, Phys. Rev. B 30, 1477 (1984).

[48] M. S. S. Challa, D. P. Landau, and K. Binder, Phys. Rev. B 34, 1841 (1986). 
Table 1. Reduced first-neighbour interaction parameters.

\begin{tabular}{ccccc}
\hline$\varepsilon_{i j}^{\mathrm{FN}}$ & $\mathrm{S}_{\mathrm{I}}^{-}$ & $\mathrm{S}_{\mathrm{I}}$ & $\mathrm{S}_{\mathrm{N}}$ & $\mathrm{TPA}$ \\
\hline $\mathrm{S}_{\mathrm{I}}^{-}$ & 0 & 0 & 0 & 5 \\
$\mathrm{~S}_{\mathrm{I}}$ & 0 & 0 & -0.8 & 5 \\
$\mathrm{~S}_{\mathrm{N}}$ & 0 & -0.8 & -1 & 5 \\
$\mathrm{TPA}$ & 5 & 5 & 5 & 5 \\
\hline
\end{tabular}

Table 2. Reduced second-neighbour interaction parameters.

\begin{tabular}{ccccc}
\hline$\varepsilon_{i j}^{\mathrm{SN}}$ & $\mathrm{S}_{\mathrm{I}}^{-}$ & $\mathrm{S}_{\mathrm{I}}$ & $\mathrm{S}_{\mathrm{N}}$ & $\mathrm{TPA}$ \\
\hline $\mathrm{S}_{\mathrm{I}}^{-}$ & 0 & 0 & 0 & -2 \\
$\mathrm{~S}_{\mathrm{I}}$ & 0 & 0 & 0 & 0 \\
$\mathrm{~S}_{\mathrm{N}}$ & 0 & 0 & 0 & 0 \\
$\mathrm{TPA}$ & -2 & 0 & 0 & 5 \\
\hline
\end{tabular}




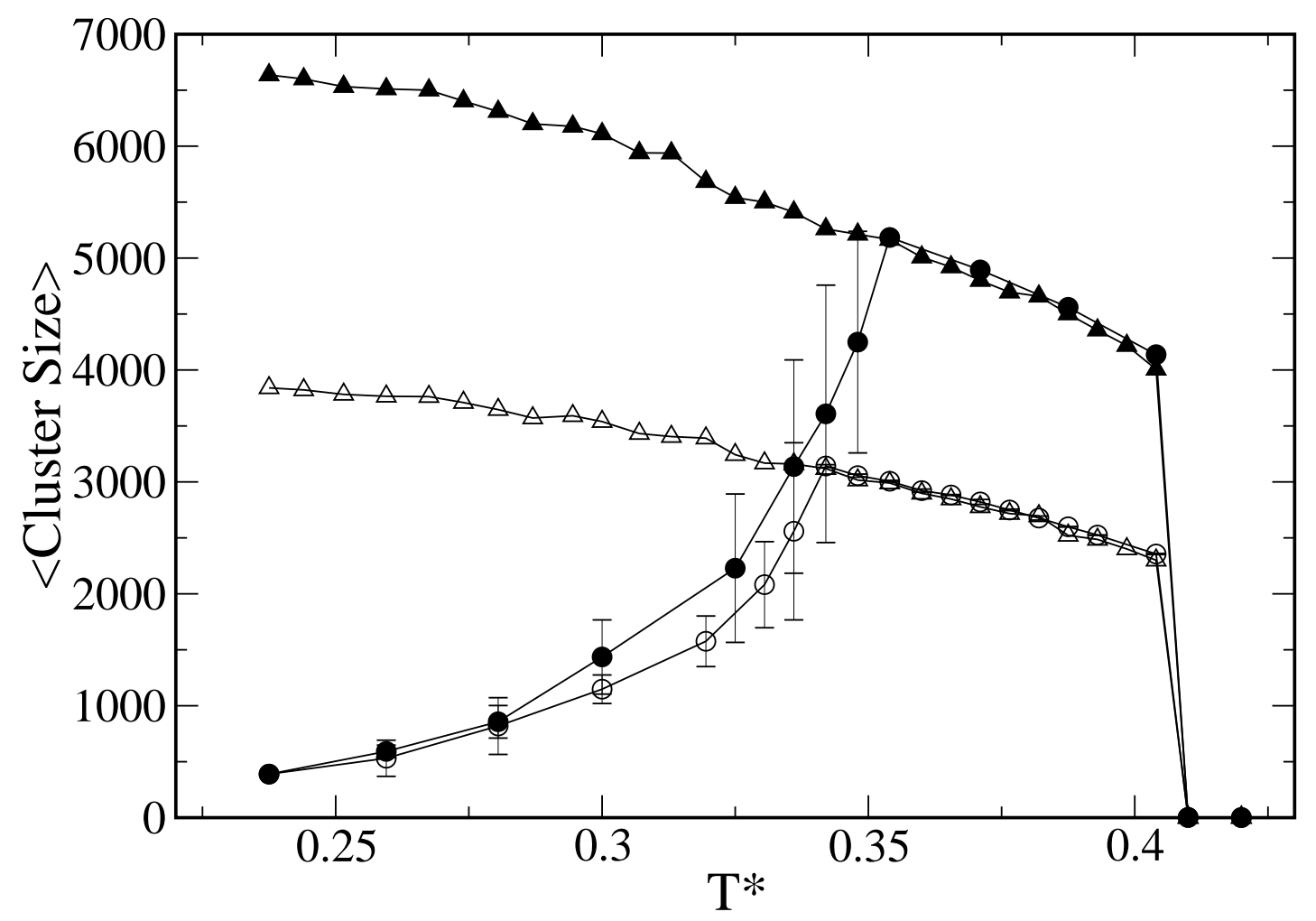

Figure 1. Average cluster size as a function of reduced temperature. Circles are results of $N V T$ simulations, averaged over five independent runs, while triangles are results of PT simulations. Open symbols correspond to a lattice of $L=100$ and closed symbols are for $L=120$. Lines are a guide to the eye. 


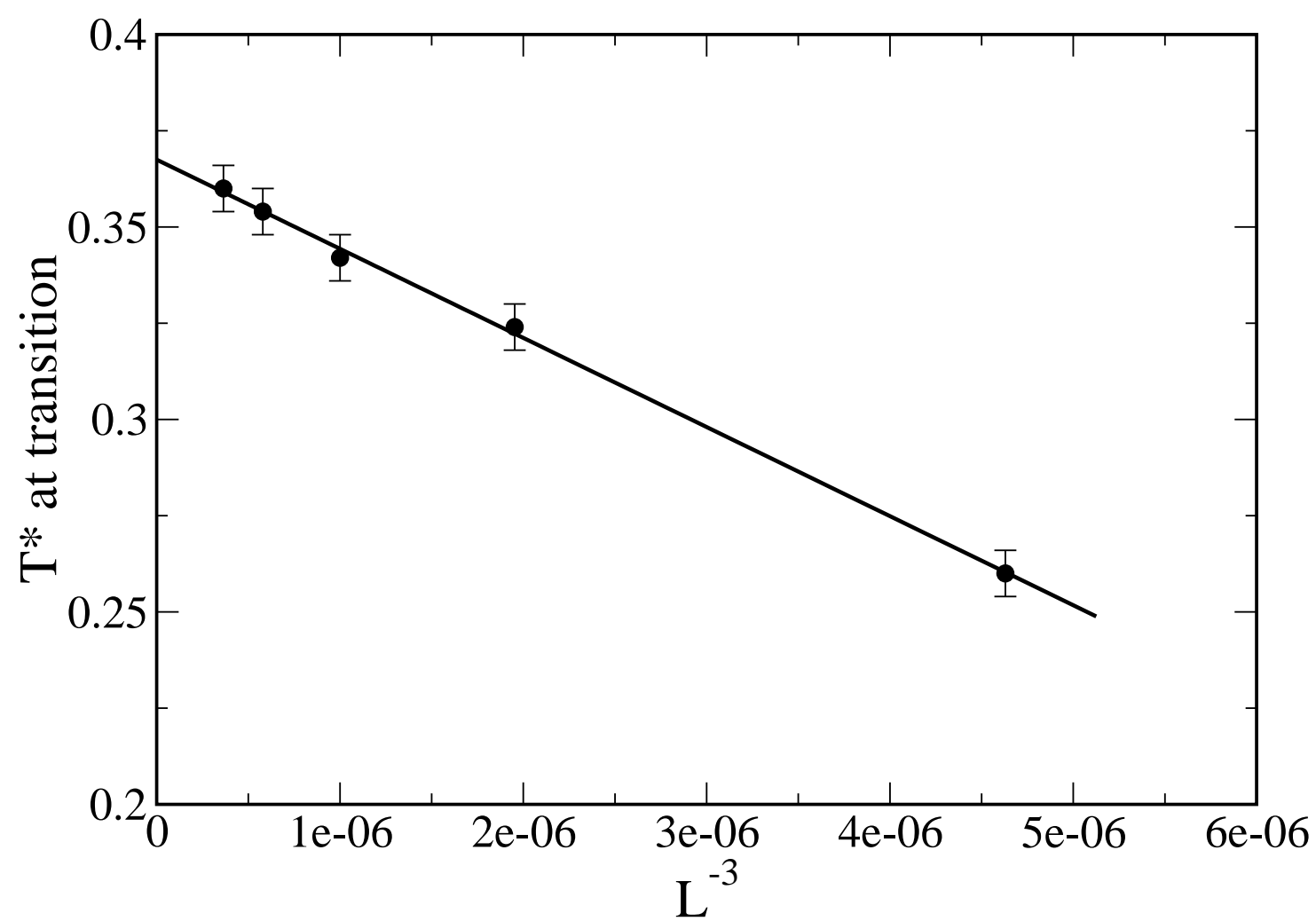

Figure 2. Transition temperature from nanoparticles to bulk, calculated from five independent $N V T$ simulations, as a function of inverse system volume. The line is a linear fit to the data points, extrapolated to infinite lattice size. 

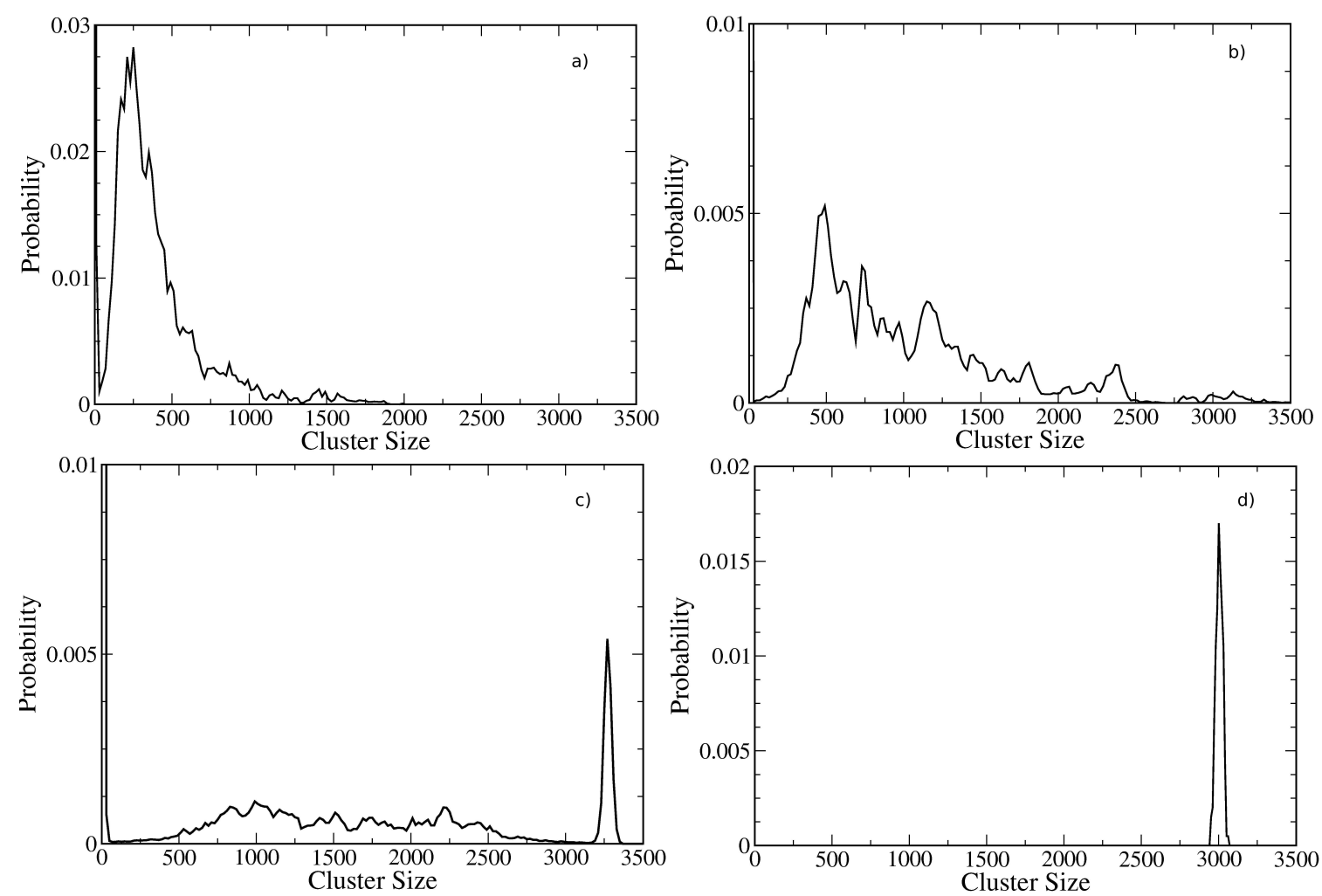

Figure 3. Cluster size distributions, averaged over $60 \mathrm{NVT}$ runs, for $L=100$ and four different temperatures: a) $T^{*}=0.24$; b) $T^{*}=0.30$; c) $T^{*}=0.33$; d) $T^{*}=0.35$. 


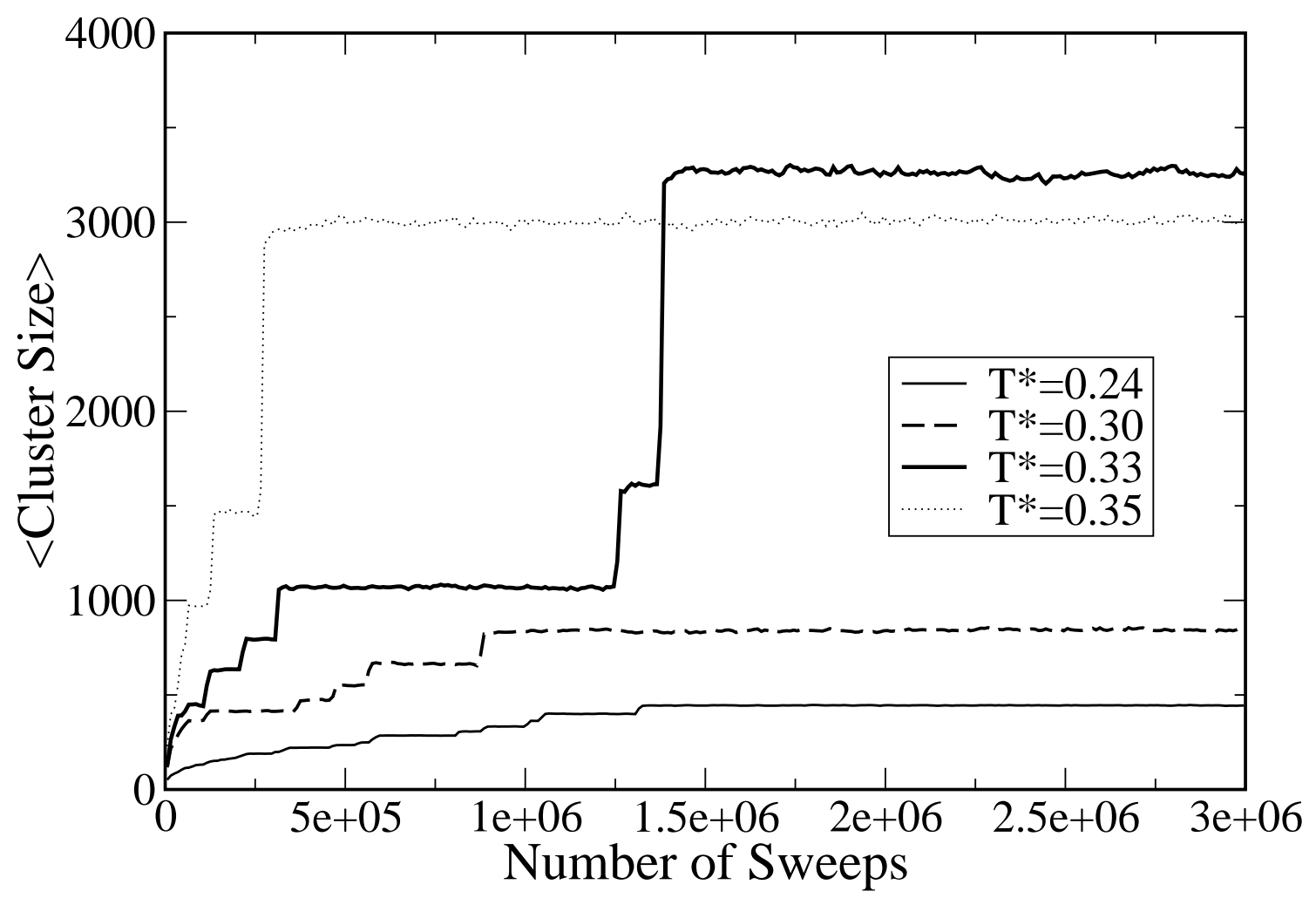

Figure 4. Evolution of average cluster size throughout individual $N V T$ runs, for $L=100$ and four different temperatures: $T^{*}=0.24$ (thin solid line); $T^{*}=0.30\left(\right.$ dashed line); $T^{*}=0.33$ (thick solid line); $T^{*}=0.35$ (dotted line) 

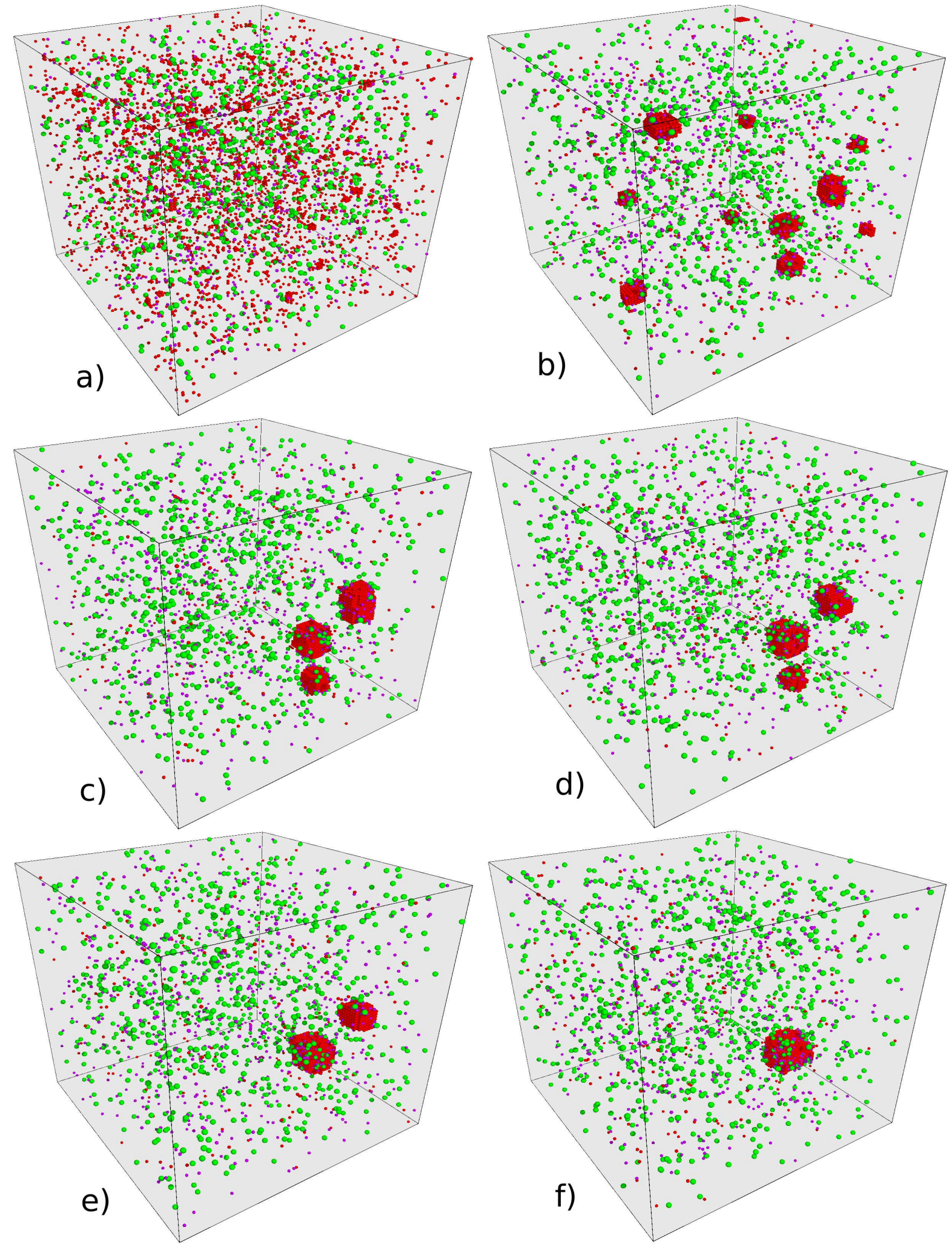

Figure 5. Snapshots of configurations obtained at different stages of an $N V T$ run at $T^{*}=0.33$ and $L=100$, corresponding to the thick solid line in figure 4: a) $10^{3}$ sweeps; b) $10^{4}$ sweeps; c) $4 \times 10^{5}$ sweeps; d) $1.2 \times 10^{6}$ sweeps; e) $1.3 \times 10^{6}$ sweeps; f) $1.4 \times 10^{6}$ sweeps. Red spheres are $S_{N}$ molecules, purple spheres are $S_{I}$ molecules and green spheres are TPA cations. Panels c) and d) show metastable nanoparticles, while panel f) shows bulk solid. 